NASA-CR-203338

\title{
Multiwire thermocouples in reversing flow
}

\section{J. Forney}

School of Chemical Engineering, Georgia Institute of Technology, Atlanta, Georgia 30332

G. C. Fralick

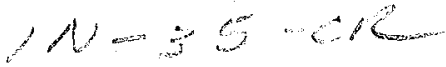

NASA-Lewis. Research Center Cleveland, Ohio 44135

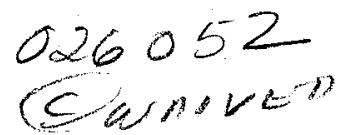

(Received 3 January 1995; accepted for publication 22 June 1995)

Measurements are recorded for multiwire thermocouples consisting of either two or three wires of unequal diameters. Signals from the multiwire probe are recorded for a reversing gas flow with both a periodic temperature and time constant fluctuation. It is demonstrated that the reconstructed signal from the multiwire thermocouple requires no compensation provided $\omega / \omega_{1}<2.3$ for two wires or $\omega / \omega_{1}<3.6$ for three wires where $\omega_{1}(=2 \pi f)$ is the natural frequency of the smaller wire based on the maximum gas velocity. The latter results were possible provided Fourier transformed data from the wires were used and knowledge of the gas velocity phase angle was available. (C) 1995 American Institute of Physics.

\section{INTRODUCTION}

The advantages of thermocouples are their low cost, reliability, and simplicity since they do not require optical access or elaborate support electronics. The design of a thermocouple represents a compromise between accuracy, ruggedness, and rapidity of response. Although thermocouples are suitable for the measurement of high-frequency temperature fluctuations $(<1 \mathrm{KHz})$ in a flowing gas or liquid, the measured signal must be compensated since the frequency of the time-dependent fluid temperature can be much higher than the natural frequency of the thermocouple probe. ${ }^{1}$ Moreover, use of a single wire thermocouple requires knowledge of the fluid velocity (normally assumed constant) and fluid properties (e.g., viscosity, density, etc.) to determine the convective heat transfer coefficient of the wire and its natural frequency.

The present article describes the performance of novel two or three-wire thermocouples with unequal wire diameters for use in unsteady or irregular flows of varying velocity. In this case, the time constant or natural frequency of the thermocouple wire is time dependent and the normal procedure for compensation of high-frequency signals is impractical. Previous work ${ }^{2-4}$ indicated the usefulness of the concept in constant velocity flows, where it was demonstrated that no compensation was required nor any knowledge of the fluid velocity or properties over a useful range of fluid temperature frequencies. In the present article, the multiwire thermocouple has been tested in a reversing flow field. The results of experimental measurements are presented along with the suggested procedure for the reduction of the data from the multiwire thermocouple, as shown in Fig. 1.

\section{THEORY}

Unsteady fluid motion develops in a variety of circumstances. For example, surfaces of discontinuity behind sharp edges such as airfoils and adverse pressure gradients on blunt objects and in sharp corners lead to flow separation and the formation of recirculating eddies. ${ }^{5}$ Other examples of irregu- lar fluid motion are (a) cellular vorticies in stratified fluids or (b) oscillating flows in the exhaust from certain engines (e.g., stirling, etc.).

\section{A. Natural frequency of single wire}

The conservation of energy for a single wire is

$\frac{d T}{d t}=\omega_{n}\left(T_{g}-T\right)$,

where the natural frequency in radians/s

$$
\omega_{n}=\frac{4 h}{\rho c D} \text {. }
$$

Here:

$$
\begin{aligned}
& \omega_{n}=\text { natural frequency }\left(\mathrm{s}^{-1}\right), \\
& T=\text { temperature of the thermocouple }\left({ }^{\circ} \mathrm{K}\right), \\
& T_{g}=\text { temperature of the } \operatorname{gas}\left({ }^{\circ} \mathrm{K}\right), \\
& t=\operatorname{time}(\mathrm{s}), \\
& \rho=\text { wire density }\left(\mathrm{kg} / \mathrm{m}^{3}\right), \\
& c=\text { heat capacity of } \operatorname{wire}\left(\mathrm{J} / \mathrm{kg}-{ }^{\circ} \mathrm{K}\right), \\
& h=\text { heat transfer coefficient }\left(\mathrm{W} / \mathrm{m}^{2}-{ }^{\circ} \mathrm{K}\right), \\
& D=\text { wire diameter }(m) .
\end{aligned}
$$

It is convenient to rewrite the heat transfer coefficient in terms of the Nusselt number

$$
h=\frac{k_{g}}{D} \mathrm{Nu}
$$

where

$\mathrm{kg}=$ thermal conductivity of the $\operatorname{gas}\left(\mathrm{W} / \mathrm{m}-{ }^{\circ} \mathrm{K}\right)$

$\mathrm{Nu}=$ Nusselt number.

Thus, the natural frequency in Eq. (2) becomes

$$
\omega_{n}=\frac{4 h_{g} \mathrm{Nu}}{(\rho c) D^{2}},
$$




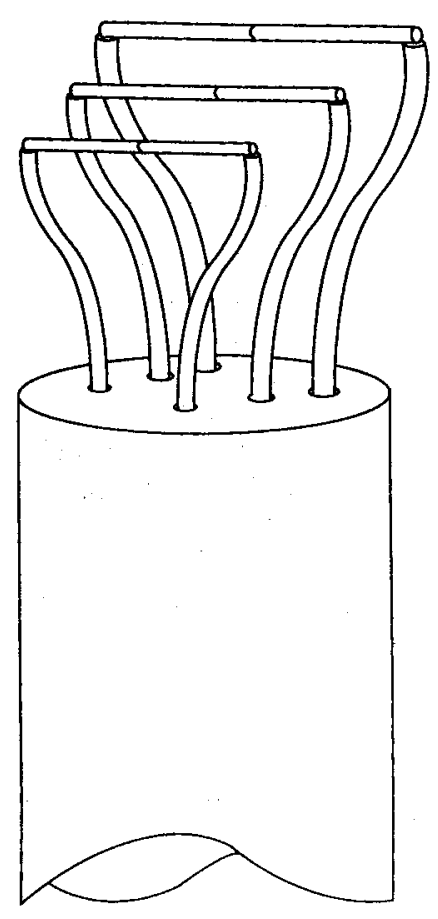

FIG. 1. Schematic of a three-wire thermocouple. Wire diameters are 2,4 , and 8 mils.

where $^{6}$

$$
\mathrm{Nu}=C_{0} \operatorname{Re}^{m} \operatorname{Pr}^{1 / 3}
$$

and .

$$
\begin{aligned}
& \operatorname{Re}=\frac{|V(t)| D}{\nu_{g}} \\
& \operatorname{Pr}=\frac{\mu c_{p}}{k_{g}}
\end{aligned}
$$

Here, $\operatorname{Re}$ and $\operatorname{Pr}$ are the Reynolds and Prandtl numbers, respectively, and

$\mu=$ viscosity of $\operatorname{gas}(\mathrm{kg} / \mathrm{m} \mathrm{s})$,

$\nu_{g}=$ kinematic viscosity of gas $\left(\mathrm{m}^{2}-\mathrm{s}^{-1}\right)$,

$V(t)=$ time dependent fluid velocity $(\mathrm{m} / \mathrm{s})$.

The natural frequency of a single wire from Eqs. (4) and (5) can now be written in the form,

$$
\omega_{n}=\frac{k_{g} C_{0} 4 \operatorname{Re}^{m} \operatorname{Pr}^{1 / 3}}{(\rho c) D^{2}}
$$

or separating the wire diameter and fluid velocity

$$
\omega_{n}=C|V|^{m} D^{m-2}
$$

where the coefficient $C$ depends on both fluid and wire parameters or

$$
C=\frac{4 k_{g} C_{0} \operatorname{Pr}^{1 / 3}}{(\rho c) \nu_{g}^{m}} .
$$

TABLE I. Three-wire diameters in Fig. 1.

\begin{tabular}{ccc}
\hline \hline$\omega_{\max }\left(\mathrm{s}^{-1}\right)$ & $D(\mathrm{~m})$ & $D(\mathrm{mil})$ \\
52.6 & $50.8 \times 10^{-6}$ & 2 \\
18.3 & $101.6 \times 10^{-6}$ & 4 \\
6.4 & $203.2 \times 10^{-6}$ & 8 \\
\hline
\end{tabular}

Substituting values corresponding to a type $K$ thermocouple and a maximum gas velocity $V_{\max }=25 \mathrm{~m} / \mathrm{s}^{-1}$ for air at standard conditions into Eqs. (7) and (8), one obtains values of the maximum natural frequency $\omega_{\max }$.

\section{B. Two wires}

The conservation of energy given by Eq. (1) for a single wire contains two unknowns: $\omega_{n}$ the natural frequency and the desired gas temperature $T_{g}$. Each additional wire of a different diameter adds an unknown natural frequency $\omega_{n}$. We also assume for a multiwire thermocouple that additional information is available concerning the ratios of natural frequencies. The latter provides a sufficient number of independent equations to determine the time-dependent gas temperature $T_{g}$.

Consider two thermocouple wires of unequal diameter $D_{1}<D_{2}$. The conservation of energy for each wire becomes

$$
\begin{aligned}
& \frac{d T_{1}}{d t}=C|V|^{m} D_{1}^{m-2}\left(T_{g}-T_{1}\right), \\
& \frac{d T_{2}}{d t}=C|V|^{m} D_{2}^{m-2}\left(T_{g}-T_{2}\right) .
\end{aligned}
$$

In principle, knowledge of the wire diameters provides an additional equation

$$
\frac{\omega_{1}}{\omega_{2}}=\left(\frac{D_{1}}{D_{2}}\right)^{m-2}
$$

representing the ratio of natural frequencies as defined by Eq. (7). Dividing Eq. (9) by Eq. (11) and solving for the gas temperature one obtains

$$
T_{g}=\frac{T_{2}\left(\frac{\dot{T}_{1}}{\dot{T}_{2}}\right)-T_{1}\left(\frac{D_{1}}{D_{2}}\right)^{m-2}}{\left(\frac{\dot{T}_{1}}{\dot{T}_{2}}\right)-\left(\frac{D_{1}}{D_{2}}\right)^{m-2}}
$$

where $\dot{T}_{1}=d T_{1} / d t$.

Our experience with multiwire thermocouples in constant flow $w^{3,4}$ and the present study indicate that construction of the gas temperature from Eq. (12), which requires the measured wire signals $T_{1}, T_{2}$ and their derivatives, is unstable and unreliable. We have found in all cases, however, that satisfactory results are obtained by taking the Fourier transform of the conservation equations and deriving the gas temperature in terms of transformed variables. For the case of unsteady flow, independent knowledge of the gas velocity is therefore required. 
Thus, taking the fast Fourier transform (FFT) of Eqs. (9) and (10) and dividing the expressions, the transform of the product of gas temperature and fluid velocity is obtained in the form

$$
\bar{T}_{g v}=\frac{\bar{T}_{2 v}\left(\frac{\bar{T}_{1}}{\bar{T}_{2}}\right)-\bar{T}_{1 v}\left(\frac{D_{1}}{D_{2}}\right)^{m-2}}{\left(\frac{\bar{T}_{1}}{\bar{T}_{2}}\right)-\left(\frac{D_{1}}{D_{2}}\right)^{m-2}},
$$

where $\bar{T}_{1 v}=\mathrm{FFT}\left(|V|^{m} T_{1}\right)$ and $\bar{T}_{g v}=\mathrm{FFT}\left(|V|^{m} T_{g}\right)$. For convenience we also define the parameter $\beta=\omega_{1} / \omega_{2}$ or

$$
\beta=\left(\frac{D_{1}}{D_{2}}\right)^{m-2}
$$

The inverse transform of $\bar{T}_{g v}$ provides the reconstructed gas temperature or

$$
T_{g}=\frac{1}{|V|^{m}} \mathrm{FFT}^{-1}\left[\bar{T}_{g v}\right] .
$$

\section{Three wires}

Consider three thermocouple wires of unequal diameters $D_{1}<D_{2}<D_{3}$ as shown in Fig. 1. The conservation expression for each wire becomes

$$
\begin{aligned}
& \frac{d T_{1}}{d t}=\omega_{1}\left(T_{g}-T_{1}\right), \\
& \frac{d T_{2}}{d t}=\omega_{2}\left(T_{g}-T_{2}\right), \\
& \frac{d T_{3}}{d t}=\omega_{3}\left(T_{g}-T_{3}\right),
\end{aligned}
$$

with the additional constraint

$$
\alpha=\frac{\omega_{2}^{2}}{\omega_{1} \omega_{3}}=\left(\frac{D_{2}^{2}}{D_{1} D_{3}}\right)^{m-2} .
$$

Taking the FFT of Eqs. (15)-(17), squaring Eq. (16) and dividing by the product of Eqs. (15) and (17), one obtains

$$
\left(\frac{\bar{T}_{2}^{2}}{\bar{T}_{1} \bar{T}_{3}}\right)=\frac{\alpha\left(\bar{T}_{g v}-\bar{T}_{2 v}\right)^{2}}{\left(\bar{T}_{g v}-\bar{T}_{1 v}\right)\left(\bar{T}_{g v}-\bar{T}_{3 v}\right)} .
$$

Substituting $r=\bar{T}_{2}^{2} / \bar{T}_{3} \bar{T}_{1}$ and solving for $\bar{T}_{g v}$, one obtains

$$
\bar{T}_{g v}=\frac{-b-\sqrt{b^{2}-4 a c}}{2 a},
$$

where

$$
\begin{aligned}
& a=r-\alpha, \\
& b=2 \alpha \bar{T}_{2 v}-r\left(\bar{T}_{1 v}+\bar{T}_{3 v}\right), \\
& c=r \bar{T}_{1 v} \bar{T}_{3 v}-\alpha \bar{T}_{2 v}^{2},
\end{aligned}
$$

and $\bar{T}_{g v}=\operatorname{FFT}\left(|V|^{m} T_{g}\right), \bar{T}_{1 v}=\mathrm{FFT}\left(|V|^{m} T_{1}\right)$. The inverse transform of $\bar{T}_{g v}$ provides the reconstructed gas temperature or

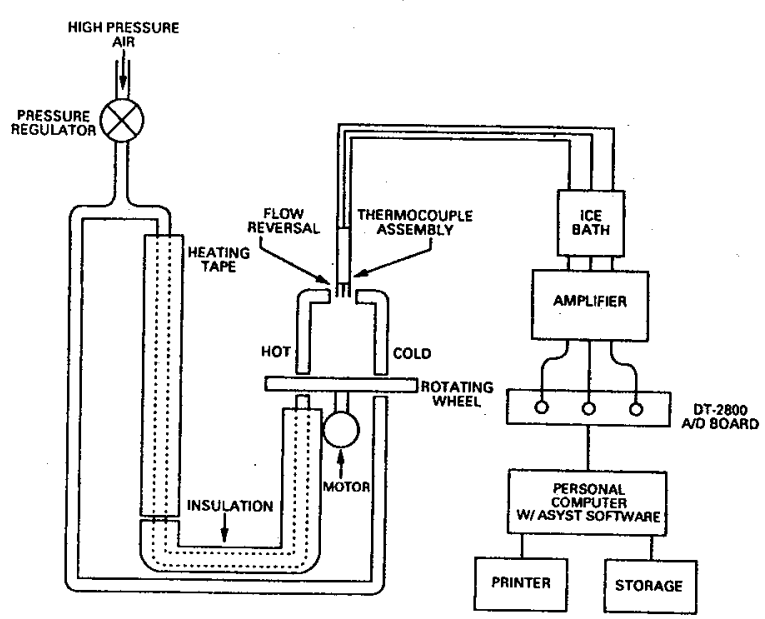

FIG. 2. Schematic of rotating wheel apparatus.

$$
T_{g}=\frac{1}{|V|^{m}} \mathrm{FFT}^{-1}\left[\bar{T}_{g v}\right]
$$

\section{EXPERIMENT}

\section{A. Apparatus}

In the present experiment, thermocouple sensors are exposed to a reversing velocity air stream $(<25 \mathrm{~m} / \mathrm{s})$ of varying temperature. In particular, the dynamic response of the thermocouple is measured for a periodic temperature profile of varying frequency. A rotating wheel configuration is used to deliver the test air stream to the proposed sensors. A similar experimental apparatus was described in detail by Elmore et al. $^{7}$ and Forney et al. ${ }^{8}$

A schematic of the rotating wheel apparatus used in the present experiment is shown in Fig. 2. As the wheel rotates, holes pass the two air supply tubes ( $3 / 4$ in. i.d. copper) that allow slugs of hot $\left(\sim 55^{\circ} \mathrm{C}\right)$ and cold $\left(\sim 30^{\circ} \mathrm{C}\right)$ air to alternately enter the tube assembly mounted directly above the rotating wheel. The slugs of hot and cold air form a reversing air stream providing a periodic temperature profile covering a range of gas temperature frequencies from roughly 1 to 60 $\mathrm{Hz}$.

The analog temperature signal is digitized with a Data Translation DT-2801 A/D board mounted in an expansion slot of an IBM AT compatible computer as shown in Fig. 2. ASYST software was loaded onto the hard disk of the personal computer and this provided a flexible system for data storage, manipulation, and display. The true temperature profile of the airstream is measured with a constant current anemometer (TSI 1054-A) and sensor (1210-T1.5).

The three type $K$ thermocouple wires were of diameter $D_{1}=50.8 \mu \mathrm{m}(2 \mathrm{mil}), D_{2}=101.6 \mu \mathrm{m}$, and $D_{3}=203.2$ $\mu \mathrm{m}$, as shown in Fig. 1. The thermocouple junctions were fabricated by the Research Instrumentation Branch at the NASA Lewis Center. The wires were cut with a razor blade to produce a flat edge perpendicular to the wire axis. The wire segments were then mounted in a fixture so that the two faces of the junction were held together by springs. The junction was then formed by laser heating. The laser used was a 


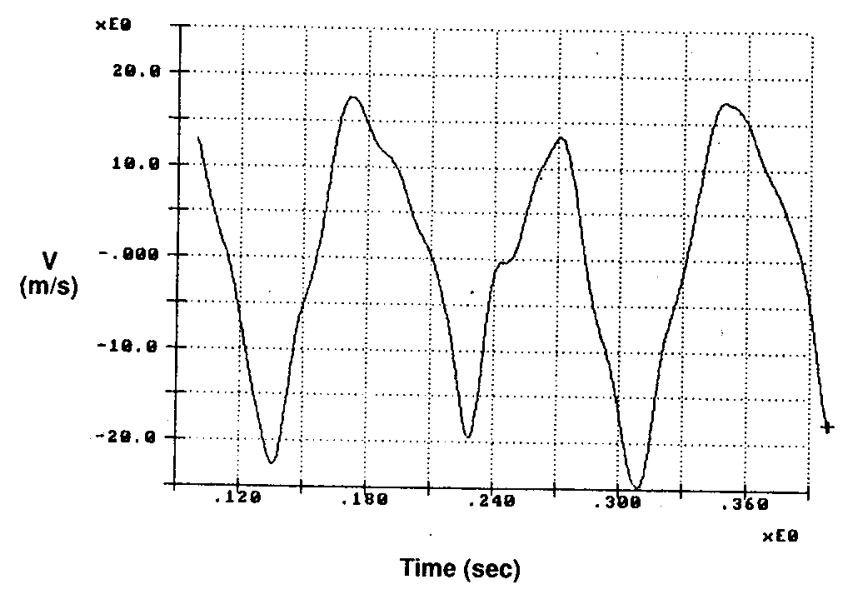

FIG. 3. Velocity profile for reversing flow. Frequency of reversing flow $f=10 \mathrm{~Hz}$.

KORAD model KWD Nd: YAG laser, operating at a wavelength of $1060 \mathrm{~nm}$. The power settings depend on the wire size and material, but for the thermocouple described in this report, a pulse duration of $4 \mathrm{~ms}$ was used, delivering a total energy of approximately $2 \mathrm{~J}$. A photograph of a junction formed in this manner is shown in an earlier article. ${ }^{8}$

\section{B. Procedure}

ASYST software was developed to acquire temperature data sequentially from the thermocouple and constant current anemometer. The data are digitized for four channels at a sampling rate of $512 \mathrm{~Hz}$ per channel for a total sample time of $0.5 \mathrm{~s}$. After data acquisition, the FFT is taken for each channel. The inverse transform of $\bar{T}_{g v}$ by the ASYST code provided a reconstruction of the true gas temperature.

\section{RESULTS AND DISCUSSION}

The velocity profile of the airstream was measured with a constant temperature anemometer. Figure 3 illustrates the velocity profile for a wheel frequency of $10 \mathrm{~Hz}$ at constant

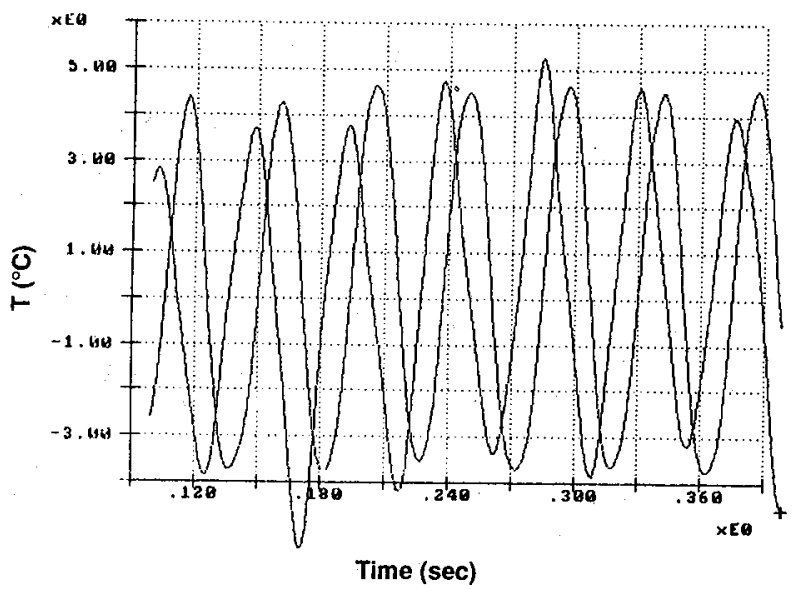

FIG. 4. Gas temperature profile compared to reconstructed temperature derived from a three-wire thermocouple. Frequency of temperature and flow reversal $f=21.5 \mathrm{~Hz}(\omega=135)$. Diameter ratio $=1,0$. The true gas temperature profile is shifted to the right for clarity.

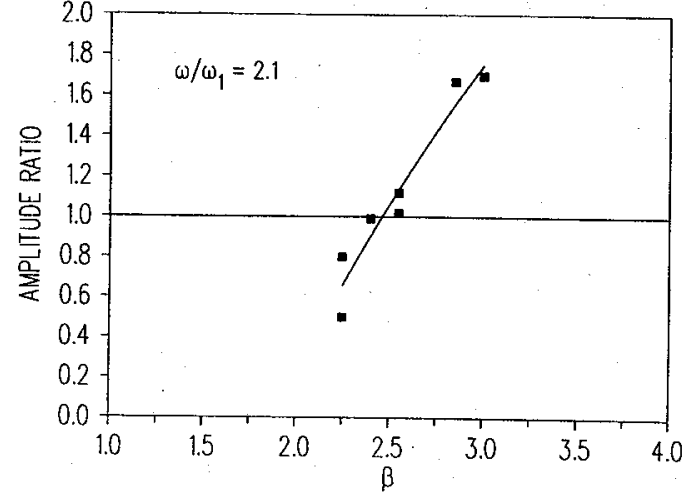

FIG. 5. Amplitude ratio of two thermocouple output-to-true gas temperature. Nominal value for diameter ratio $\beta_{0}=2.83$ corresponding to manufacturers' diameters of $D_{1}=2$ and $D_{2}=4$ mils. Optimum $\beta=2.55$.

temperature (heating tape off). For the positive velocities in Fig. 3, the gas reached a temperature of roughly $25-30{ }^{\circ} \mathrm{C}$ in the experiments below, corresponding to the cold slugs, while the negative velocity components were hot air slugs of approximately $50^{\circ} \mathrm{C}$.

In the present study, the gas velocity and true temperature were not measured simultaneously. Rather, it was assumed that the temperature measured with the anemometer was proportional to the gas velocity in the reversing flow field or $V(t) \propto T_{g}(t)$. In this case, $\left|T_{g}(t)\right|^{m}$ was substituted for $|V(t)|^{m}$ throughout the analysis, where $T_{g}(t)$ is the true gas temperature. The latter assumption was possible since $V(t)$ appears in both the numerator and the denominator of Eq. (21). Thus, the amplitude of the gas velocity was not important while the phase angle of $V(t)$ and $T_{g}(t)$ are identical.

A typical data profile constructed from Eq. (21) is illustrated in Fig. 4. The uniform profile on the right is the temperature profile measured with the anemometer. The temperature profile on the left was reconstructed with Eq. (21) assuming that the exponent $m=0.5$ in Eqs. (7) and (21) and a value of the diameter ratio $\alpha=1.0$ for the three-wire thermocouple. The phase shift provided clarity between both signals.

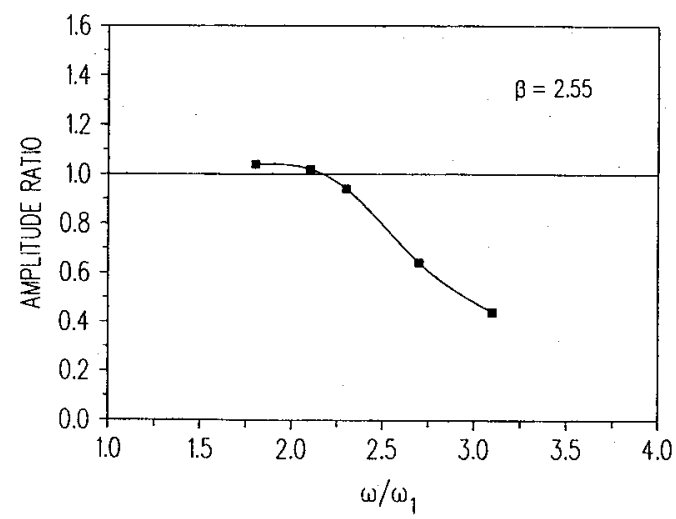

FIG. 6. Amplitude ratio of two-thermocouple output vs gas temperature frequency of small wire based on maximum gas velocity. Angular frequency $\omega_{1}=59 \mathrm{~s}^{-1}$ 


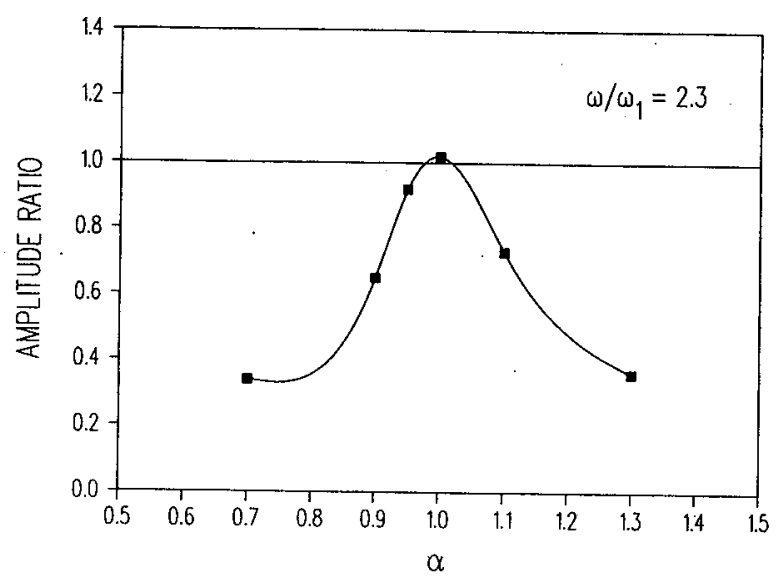

FIG. 7. Amplitude ratio of three-thermocouple output-to-true gas temperature. Nominal and optimum value for diameter ratio $\alpha_{0}=1.0$ corresponding to manufacturers' diameters of $D_{1}=2, D_{2}=4$ and $D_{3}=8$ mils.

The value of the exponent $m=0.5$ was chosen for the Reynolds number that appears in the Nusselt number correlation Eq. (5) and the wire natural frequency $\omega_{n}$ given by Eq. (7). At the maximum gas velocity of $25 \mathrm{~m} / \mathrm{s}$, the maximum Reynolds number for the three wires in the thermocouple assembly are 80,160 , and 320 , corresponding to the smallest to largest diameter wire, respectively. Since the recommended values of the exponent $m$ are 0.5 for $\operatorname{Re}>40$ and 0.4 for $1 \leqslant \operatorname{Re} \leqslant 40,{ }^{6}$ we chose a constant value $m=0.5$ in the present study.

\section{A. Two wires}

The amplitude ratio of thermocouple-to-gas temperature was determined using a two-wire combination with $D_{1}=50.8 \mu \mathrm{m}$ and $D_{2}=101.6 \mu \mathrm{m}$ from the schematic in Fig. 1. The gas temperature was reconstructed with Eq. (13) assuming $m=0.5$. The amplitude ratio was determined by averaging peak temperatures over six or eight cycles. The results are indicated in Fig. 5 as a function of the parameter $\beta=\omega_{1} / \omega_{2}=\left(D_{1} / D_{2}\right)^{-3 / 2}$. In Fig. 5 the natural frequency $\omega_{1}=59 \mathrm{~s}^{-1}$ is defined as the angular frequency at the maximum gas velocity for the small wire $D_{1}=50.8 \mu \mathrm{m}$. It is clear that the amplitude ratio is strongly affected by $\beta$ and that the optimum value is $\beta=2.55$.

It should be noted that the optimum $\beta=2.55$ differs from an expected nominal value of $\beta_{0}=2.83$, where the latter is computed from the manufacturers' wire diameters. These differences in $\beta$ can be attributed to the empirical value of the exponent $m=0.5$ chosen or to changes in wire diameter at the thermocouple junction because of the laser fabrication process.

Figure 6 illustrates amplitude attenuation as a function of gas temperature frequency for fixed $\beta=2.55$. The two-wire

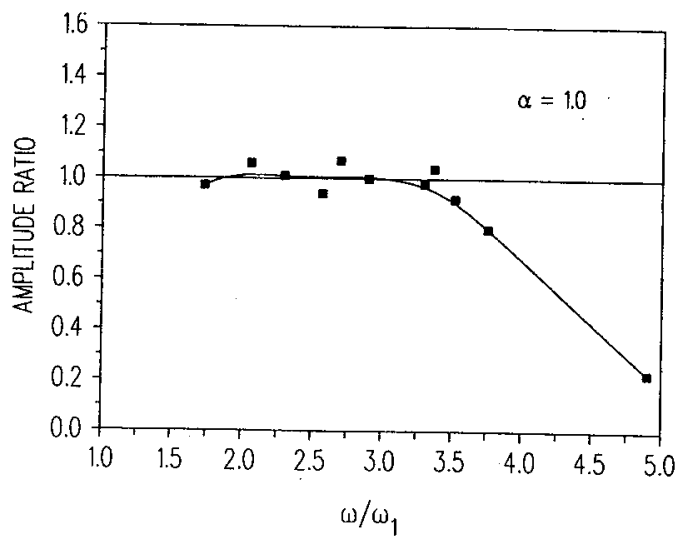

FIG. 8. Amplitude ratio of three-thermocouple output vs gas temperature frequency. Angular frequency $\omega_{1}=59 \mathrm{~s}^{-1}$.

combination requires no compensation for frequencies $\omega / \omega_{1}<2.3$ corresponding to frequencies $f<22 \mathrm{~Hz}$ in the reversing flow field since $2 \pi f_{1}=\omega_{1}=59 \mathrm{~s}^{-1}$.

\section{B. Three wires}

The amplitude ratio of thermocouple-to-gas temperature was determined for the three-wire combination as shown in the schematic of Fig. 1. The gas temperature was reconstructed with Eqs. (20) and (21) with the exponent $m=0.5$. The results are indicated in Fig. 7 as a function of the parameter $\alpha=\left(\omega_{2}^{2} / \omega_{1} \omega_{3}\right)=\left(D_{2}^{2} / D_{1} D_{3}\right)^{-3 / 2}$. It is clear that the amplitude is strongly affected by $\alpha$ and that the optimum value is $\alpha=1.0$. The latter value also corresponds to the nominal value calculated with the manufacturers' listed wire diameters, as shown in Table $\mathbf{I}$.

Figure 8 illustrates amplitude attenuation as a function of gas temperature frequency for fixed $\alpha=1.0$. The three-wire combination requires no compensation for frequencies $\omega / \omega_{1}<3.5$ corresponding to frequencies $f<34 \mathrm{~Hz}$ in the reversing flow field.

\section{ACKNOWLEDGMENTS}

The work reported herein was sponsored by a NASA cooperative agreement NCC 3-302. The authors also wish to acknowledge the support of Dr. W. D. Williams, head of the Research Sensor Technology Branch of NASA-Lewis.

${ }^{1}$ M. D. Scadron and I. Warshawski, NASA Report No. TN-2599, 1952 (unpublished).

${ }^{2}$ G. C. Fralick, Patent disclosure (25 April 1990).

${ }^{3}$ L. J. Forney and G. C. Fralick, Rev. Sci. Instrum. 65, 3252 (1994).

${ }^{4}$ L. J. Forney and G. C. Fralick, Rev. Sci. Instrum. 66, 3331 (1995).

${ }^{5}$ L. Prandl, Essentials of Fluid Dynamics (Hafner, New York, 1952).

${ }^{6}$ F. P. Incropera and D. P. DeWitt, Fundamentals of Heat and Mass Transfer, 2nd ed. (Wiley, New York, 1985).

${ }^{7}$ D. L. Elmore, W. W. Robinson, and W. B. Watkins, NASA Report No. CR-179513, 1986 (unpublished). ${ }^{8}$ L. J. Forney, E. L. Meeks, J. Ma, and G. C. Fralick, Rev. Sci. Instrum. 64,
3236 (1993). 
